\title{
Time-dependent cytotoxic drugs selectively cooperate with IL-18 for cancer chemo- immunotherapy
}

Ioannis Alagkiozidis ${ }^{1 *}$, Andrea Facciabene ${ }^{1}$, Marinos Tsiatas $^{1}$, Carmine Carpenito ${ }^{2}$, Fabian Benencia ${ }^{2}$, Sarah Adams ${ }^{1}$, Zdenka Jonak ${ }^{4}$, Carl H June ${ }^{2}$, Daniel J Powell $\mathrm{Jr}^{1,3}$ and George Coukos ${ }^{1,2}$

\begin{abstract}
Background: Time-dependent chemotherapeutic agents can selectively target tumor cells in susceptible phases of the cell cycle however a fraction of tumor cells in non-vulnerable cell cycle phases remain drug-resistant. Immunotherapy represents a promising approach to overcome the limitation of phase-specific drugs and improve their clinical efficacy. Here, we investigated the potential use of anticancer chemotherapeutic drugs in combination with IL-18, a cytokine with strong immunostimulatory properties.

Methods: Four chemotherapeutic drugs commonly used in ovarian cancer were first tested for the ability to increase the immunogenicity and killing of the murine ovarian cancer cell line ID8 in vitro. Chemotherapeutric agents with measured time-dependent immune-enhancing effects were then tested for antitumor effectiveness in vivo in combination with IL-18 immunotherapy using the ID8-Vegf ovarian cancer model.

Results: Paclitaxel or topotecan exposure alone mediated incomplete, time-dependent killing against the murine ovarian cancer cell line ID8 in vitro, whereas carboplatin or gemcitabine mediated comprehensive, dose-dependent killing. In the plateau phase of the time-dependent killing by topotecan or paclitaxel, drug-resistant ID8 cells were more immunogenic with elevated expression of MHC-I and Fas, and increased sensitivity to CTL and Fas agonistic antibody in vitro. Moreover, the antitumor effectiveness of time-dependent agents in vivo was significantly improved with the addition of IL-18 through a T cell-dependent mechanism, while the effectiveness of drugs without significant phase specificity were not.

Conclusions: Tumor immunotherapy with IL-18 can significantly augment the killing fraction of phase-specific chemotherapeutic drugs and provide survival benefit. The safety profile of IL-18 and its positive interactions with select anticancer chemotherapeutic agents strongly supports the clinical investigation of this combinatorial approach.
\end{abstract}

\section{Background}

Although chemotherapy is the treatment of choice for many types of cancer, it is rarely curative in most solid tumors. Immune therapy represents a potentially attractive approach to increase the efficacy of chemotherapy by targeting cancer cells that escape chemotherapy. However, it has been unclear to date whether any chemotherapy drugs are more suitable than others for such

\footnotetext{
*Correspondence: loannis.Alagkiozidis@uth.tmc.edu

'Ovarian Cancer Research Center, School of Medicine, University of

Pennsylvania, Philadelphia, USA

Full list of author information is available at the end of the article
}

combinations, and empirical use has produced mixed results. For example, although higher objective response and disease control rates, along with elevated frequencies of cytolytic tumor antigen-specific $\mathrm{T}$ cells, were seen in patients with metastatic colorectal carcinoma receiving polychemotherapy with gemcitabine plus oxaliplatin, fluorouracil, and folinic acid (FOLFOX-4) followed by granulocyte-macrophage colony-stimulating factor (GM-CSF) and low-dose interleukin-2 (IL-2) [1], addition of IL-2 and interferon-alpha2b did not increase the efficacy of cisplatin, vindesine and dacarbazine in melanoma patients [2]. Thus, understanding the

\section{Ciomed Central}


mechanisms underpinning positive chemo-immunotherapy interactions is a critical task for the development of effective cancer therapy.

Previous reports have suggested that the exposure of tumor cells to chemotherapeutic drugs can sensitize them to immune effector cells [3-6]. Theoretically, to achieve synergy with immune therapy and increased tumor killing, chemotherapy should sensitize to immune killing tumor cells that are destined to survive chemotherapy. Depending on their mechanism of action, the efficacy of chemotherapy drugs may be influenced markedly by the time of exposure (phase-specific or time-dependent drugs) or by the dose that can be administered (phase-nonspecific or dose-dependent drugs). The efficacy of phase-specific anticancer drugs is timedependent, as only a fraction of tumor cells are in appropriate cell cycle phase for chemotherapy-mediated killing at any given time. Thus, a fraction of tumor cells remains alive following administration of each chemotherapy dose and can eventually repopulate the tumor following completion of chemotherapy [7-10]. We hypothesized that because of this property, timedependent chemotherapy drugs are more likely to benefit from combination with immune therapy.

Interleukin 18 (IL-18) is a pleiotropic cytokine, originally described as interferon (IFN) $-\gamma$ inducing factor, that can mediate immunostimulatory effects on immune cells of the adaptive and innate immune system [11]. Its multiple immunologic activities include the induction of IFN- $\gamma$, TNF- $\alpha$, IL- $1 \alpha$, and GM-CSF production; augmentation of natural killer (NK) cell cytotoxicity; and promotion of Th1 differentiation of naive $\mathrm{T}$ cells. These features render IL-18 an interesting candidate for tumor immunotherapy. As a single agent, IL-18 was shown to elicit anti-tumor reactivity when administered at high doses in mice with established tumors [12]. The immunostimulatory activity of IL-18 in vivo has been demonstrated in non-human primates [13] and humans [14]. In phase I clinical evaluation, recombinant human ( $\mathrm{rh}$ ) IL-18 was safely administered as monotherapy to 28 patients with solid tumors, with minimal dose-limiting toxicities and two partial tumor responses [14]. Toxicity has generally been mild to moderate even with repeat administration and a maximum tolerated dose has not been reached to date [15]. IL-18 enhanced activation of peripheral blood $\mathrm{CD}^{+} \mathrm{T}$ cells, NK cells and monocytes and induced a transient increase in the frequency and expression level of Fas ligand (FasL) in peripheral blood $\mathrm{CD}^{+} \mathrm{T}$ cells and NK cells [15]. The relatively minor toxicity of rhIL-18, compared with other immunostimulatory cytokines that have undergone clinical development, is remarkable and renders IL-18 a well suited drug for combinatorial approaches with chemotherapy.
In the current study, we characterized the immune effects on tumor cells of four common anticancer chemotherapy drugs utilized in ovarian cancer and other solid tumors, two phase-specific (time-dependent) drugs, paclitaxel and topotecan, and two phase-nonspecific (dose-dependent) drugs, gemcitabine and carboplatin. Both paclitaxel and topotecan exert their actions on dividing cells, acting as phase-specific chemotherapeutic drugs. Paclitaxel inhibits the dissolution of microtubules, enhances tubulin polymerization and produces a block in the metaphase of mitosis, leading to growth inhibition and cell apoptosis [16]. Topotecan, a topoisomerase I inhibitor, stabilizes the covalent complex of enzyme and strand-cleaved DNA, which is an intermediate of the catalytic mechanism, thereby inducing breaks in the protein-associated DNA single-strands, resulting in cell death [17]. Carboplatin is a classic cycle phase non-specific drug [18]. The main mechanism of action of gemcitabine is inhibition of DNA synthesis. The killing effects of gemcitabine are however not confined to the S-phase of the cell cycle and the drug is equally effective against confluent cells and cells in log-phase growth [19]. Incorporation of gemcitabine into RNA is another action, which is time- and concentration-dependent and leads to inhibition of RNA synthesis. In human tumor cell lines displaying different degrees of resistance to gemcitabine, sensitivity to this drug was related to differences in RNA incorporation [20]. Moreover, several metabolites of gemcitabine can inhibit various enzymes, leading to self-potentation of gemcitabine action [21]. Thus, the overall mechanism of action of gemcitabine is phase non-specific.

Because the effect of immune therapy becomes clinically relevant only if immune mechanisms target the tumor fraction surviving chemotherapy, we focused on the fate of tumor cells escaping direct killing by chemotherapy. We hypothesized that these cells are sensitized to immune therapy, which enables a cooperation between immunotherapy and time-dependent (phasespecific) drugs. Thus, we hypothesized that among chemotherapy drugs, time-dependent (phase-specific) drugs are more likely to benefit from IL-18 therapy combination. We investigated these interactions in a mouse model of ovarian cancer [22]. IL-18 alone had a modest antitumor effect, while it exhibited positive interaction with select chemotherapeutic drugs, improving their therapeutic effect in vivo. Chemotherapeutic agents upregulated immune molecules in tumor cells and sensitized them to immune-mediated killing. Importantly, this effect was translated to a significant increase in total killing fraction and better outcome only for timedependent drugs. Interestingly, combination of IL-18 with dose-dependent drugs did not increase their efficacy in vivo. In this study however we sought to mainly 
explore the ability of those drugs to render tumor cells more susceptible to immunotherapy with IL-18, and specifically focused on effector mechanisms, and not the drugs' overall effects on the immune system. Moreover, our findings indicate for the first time that the difference of chemotherapeutic drugs in their ability to interact with immunotherapy might be attributed to their mechanism of action directly on the tumor cell. These data suggest that tumor immunotherapy with IL-18 may potentiate the antitumor effect selectively of timedependent chemotherapeutics used in various types of cancer.

\section{Methods}

\section{Cell Culture and Reagents}

ID8 ovarian cancer cells were generously donated by Drs. Kathy Robby and Paul Terranova (Kansas University) [23]. The development and characterization of ID8Vegf cell line was described elsewhere in detail [22]. The development and characterization of the ID8-E6E7 cell line transfected to stably express the E6 and E7 genes of the human papilloma virus was described elsewhere in detail [24]. Briefly, ID8 cells were transduced with the retroviral vector LXSN16E6E7 (American Type Culture Collection, Rockville, MD, donated by Dr. D. Galloway), which encodes the HPV16 E6 and E7 genes, as well as the neomycin phosphotransferase gene. The PA317 cell line was used to generate the retroviral vectors as previously described [25]. Selection of ID8 cells transduced with E6 and E7 (ID8-E6/7) or ID8 cells transduced with a control retroviral vector (LXSN) was achieved under neomycin pressure $(1 \mathrm{mg} / \mathrm{ml})$. ID8, ID8E6E7 and ID8-Vegf cells were maintained in DMEM media (Invitrogen, Carlsbad, CA) supplemented with $10 \%$ fetal bovine serum (FBS), $100 \mathrm{U} / \mathrm{ml}$ penicillin, and $100 \mu \mathrm{g} / \mathrm{ml}$ streptomycin (Roche, Indianapolis, IN) in a $5 \% \mathrm{CO}_{2}$ atmosphere at $37^{\circ} \mathrm{C}$. ID8-Vegf cells were used for in vivo experiments. ID8 cells were used for in vitro and flow cytometry experiments. ID8-E6E7 cells were used as target cells for cytotoxicity assays. All reagents were from Sigma unless stated otherwise.

\section{Mice and Treatments}

Eight to sixteen week old female C57BL/6 mice and C57BL/6 severe combined immunodeficient (SCID) mice (Charles River Laboratories, Wilmington, MA) were used in protocols approved by the Institutional Review Board of the University of Pennsylvania. Mice were treated with intraperitoneal (i.p.) bolus injections of chemotherapy drugs or PBS (controls) as follows: Carboplatin, $20 \mathrm{mg} / \mathrm{kg}$ in $0.2 \mathrm{ml} 0.9 \%$ saline, four weekly doses; paclitaxel, $15 \mathrm{mg} / \mathrm{kg}$ in $0.3 \mathrm{ml} 0.9 \%$ saline, four weekly doses; topotecan, $2.5 \mathrm{mg} / \mathrm{kg}$ in $0.2 \mathrm{ml} 0.9 \%$ saline, every 5 days (total five doses); and gemcitabine,
$25 \mathrm{mg} / \mathrm{kg}$ in $0.2 \mathrm{ml} 0.9 \%$ saline, every 3 days (total five doses). Chemotherapy doses were approximately onefourth to one-sixth of the respective maximally tolerated dose (MTD) for mice [26-30]. Recombinant murine (rm)IL-18 (GlaxoSmithKline) was given s.c daily for 40 days at $10 \mu \mathrm{g} /$ mouse in $90 \mu \mathrm{l} \mathrm{PBS}$. Control mice received s.c. daily injections of PBS $(100 \mu \mathrm{l})$ alone. Chemotherapy administration was started 8 days after tumor inoculation and IL-18 treatment was started two days later.

\section{Tumors}

Orthotopic intraperitoneal (i.p.) tumors were generated by inoculating intraperitoneally $5 \times 10^{6}$ ID8-Vegf cells suspended in $250 \mu \mathrm{l}$ PBS. Mice were weighed weekly starting two weeks after tumor inoculation. Ascites volume measurement was a terminal procedure and it was done when mice reached 35 grams of weight. When we needed week-to-week measurements of ascites accumulation, we used weight increase as surrogate. Solid subcutaneous tumors were generated by inoculating $10^{7}$ ID8-Vegf cells suspended in $250 \mu \mathrm{l}$ PBS and mixed with an equal volume of cold Matrigel into the flanks of mice. Tumors were detectable two weeks later and tumor size was measured weekly thereafter using a Vernier caliper. Since studies have shown that tumor weight is the most consistent and reproducible reflection of tumor volume, especially in small tumors [31], tumor volumes were calculated by the formula $\mathrm{V}=1 / 2(\mathrm{~L} \times \mathrm{W})^{2}$, where $\mathrm{L}$ is length (longest dimension) and $\mathrm{W}$ is width (shortest dimension). Experiments were terminated when control tumors reached the size of 600 to 800 $\mathrm{mm}^{3}$; all groups were euthanized, the tumors were excised and weighed.

\section{In vitro treatment of tumor cells}

For measurement of chemotherapy sensitivity in vitro, ID8 tumor cells $\left(1.3 \times 10^{5} /\right.$ well $)$ were exposed to cytotoxic agents for 6 hours in various concentrations: Topotecan $(0,0.1,0.3,1,3,10$, and $30 \mu \mathrm{g} / \mathrm{ml})$, paclitaxel $(0,0.03,0.1,0.3,1,3$, and $10 \mu \mathrm{g} / \mathrm{ml})$, gemcitabine $(0$, $0.02,0.06,2,6,20$, and $60 \mu \mathrm{g} / \mathrm{ml})$, carboplatin $(0,30$, $100,300,1000$, and $3000 \mu \mathrm{g} / \mathrm{ml})$ and to sodium azide $(0$, $0.1,0.3,1,3$, and $10 \mu \mathrm{g} / \mathrm{ml})$. At 6 hours the drug containing media was removed, cells were washed twice with PBS and cultured in drug free media for another 42 hours. Tumor cells were then washed twice with PBS, thrypsinized and counted. Non-viable cells were excluded with Trypan Blue staining. For Fas-mediated killing, we used the anti-Fas agonistic antibody, Jo2 (0.2ug.mL dose, BD PharMingen). Protein G $(2 \mu \mathrm{g} / \mathrm{ml}$; Biovision) was used to maximize cross-linking of the primary antibody. The antibody was added 24 hours before cell harvesting and counting. Control wells 
consisted of tumor cells incubated with isotype-matched antibody and protein G. ID8 cell survival fractions after exposure to different doses of drugs with or without exposure to Fas agonistic antibody were plotted against the drug concentrations and the resulting data set was fit to a logistic dose-response function using Origin7 software (OriginLab Corporation). $\mathrm{IC}_{50}$ values were obtained from the fit parameters that achieved the lowest $x^{2}$ value.

\section{Flow cytometry analysis}

Cell surface staining of mouse splenocytes was performed with FITC-labeled anti-CD69 mAb, PE-labeled anti- $\mathrm{CD} 4 \mathrm{mAB}, \mathrm{Pe}-\mathrm{Cy} 7$-labeled anti-CD3 mAB, PercPlabeled-anti-CD8 $\mathrm{mAb}$. For the cell surface staining of ID8 tumor cells anti-MHC-I ( $\mathrm{H}-2 \mathrm{~Kb} / \mathrm{H}-2 \mathrm{Db})$ biotinylated $\mathrm{mAb}$ and anti-Fas PE-labeled $\mathrm{mAb}$ were used. Secondary Ab was streptavidin-APC labeled. All mAb were purchased from BD PharMingen. Cell fluorescence was analyzed and compared with the appropriate isotypematched controls (BD PharMingen) with a FACSCanto cytometer and Flow Jo software.

\section{Apoptosis detection}

Detection of apoptotic cells was carried out with the TACS Annexin V-FITC apoptosis detection system (R\&D Systems) which uses annexinV-fluorescein isothiocyanate (FITC) conjugates for flow cytometry.

\section{Lymphocyte cytotoxicity assay}

ID8-E7 cells were prepared for use as targets in a colorimetric non-radioactive cytotoxicity assay measuring lactate dehydrogenase (Promega). Target cells were exposed to topotecan or paclitaxel for 6 hours, washed and cultured for an additional 42 hours. The target cells $\left(12 \times 10^{3}\right.$ cells/well $)$ were coincubated with splenocytes from vaccinated donor mice (see below) at various effector:target (E:T) cell ratios, in a final volume of $200 \mu \mathrm{l}$ RPMI supplemented with $10 \%$ FBS $(100 \mathrm{U} / \mathrm{ml}$ penicillin and $100 \mu \mathrm{g} / \mathrm{ml}$ streptomycin) for 4 hours at $37^{\circ} \mathrm{C}$ with $5 \% \mathrm{CO}_{2}$. For the generation of effector cells, eight to sixteen week old C57BL6 mice were vaccinated twice, one week apart, with LLO-E7 DNA vaccine [32] encoding the E7 peptide and the Listeria Listeriolysin O (LLO) adjuvant (kindly provided by Dr. Yvonne Patterson), and one month later they were challenged with 5 $\times 10^{5} \mathrm{TC}-1$ cells (which express E7) injected s.c in the flank as boost, which increases significantly the frequency of E7-reactive T cells in the spleen. Two weeks later, mice were sacrificed, spleens were harvested, splenocytes were isolated and stimulated in vitro for 7 days with $30 \mathrm{IU} / \mathrm{ml} \mathrm{IL-2}$ and $8 \mu \mathrm{g} / \mathrm{ml} \mathrm{E7} \mathrm{peptide} \mathrm{in} \mathrm{RPMI}$ media (Invitrogen) supplemented with $10 \%$ fetal bovine serum (FBS), $100 \mathrm{U} / \mathrm{ml}$ penicillin, and $100 \mathrm{mu} \mathrm{\mu g} / \mathrm{ml}$ streptomycin (Roche) in a $5 \% \mathrm{CO} 2$ atmosphere at $37^{\circ} \mathrm{C}$. The percent cytotoxicity was calculated with the formula:

$\%$ Cytotoxicity $=\frac{[\text { Experimental }- \text { Effector spontaneous }- \text { Target spontaneous }] \times 100}{[\text { Target maximum }- \text { Target spontaneous }] .}$

\section{Statistical methods}

A two-tailed Student's t-test was used for betweengroup comparisons of in vitro and flow cytometry data. Tumor growth curves depict median and the error bars interquartile range (25\%-75\%). Differences were considered significant at the level of $p<0.05$ (Student's test). The Kaplan-Meier method was used to estimate survival curves from animal studies. Survival curves were compared with the Wilcoxon statistic.

\section{Results}

ID8 tumor cell killing by topotecan and paclitaxel is phase-dependent

Chemotherapeutic drugs commonly employed against solid tumors such as ovarian cancer, include carboplatin, paclitaxel, topotecan and gemcitabine. Among these, topotecan and paclitaxel are known to be cycle phasespecific and thus their effect is time- rather than dosedependent. The sensitivity of ID8 mouse ovarian cancer cells to the above drugs was tested in vitro. To mimic exposure in vivo, where the half life of chemotherapy drugs is short [33,34], cells were exposed to drug for 6 hours and then were followed for an additional 42 hours. Cell count data at 48 hours were fitted to a logistic regression dose-response curve (Figure $1 \mathrm{~A}$ ). The $\mathrm{IC}_{50}$ - as calculated from these dose response curves - was used to compare killing curves of the different cytotoxic agents. ID8 cells were killed by all drugs. A characteristic of phase-specific drugs is that their cytotoxicity depends mainly on time of exposure rather than on drug concentration $[8,10]$. The survival curves of topotecan and paclitaxel reached a plateau at approximately 5fold the $\mathrm{IC}_{50}$, with a substantial fraction of cells surviving in spite of further dose increase; > 35\% of the cells survived from topotecan and $>20 \%$ of cells survived from paclitaxel. By contrast, gemcitabine and carboplatin exhibited dose-dependent, phase-nonspecific killing in vitro.

\section{Chemotherapeutic drugs induce MHC and Fas expression in tumor cells}

It has been suggested that cell damage induced by chemotherapy upregulates the expression of MHC-I or costimulatory molecules (e.g., NKG2D ligands) in tumor cells, sensitizes them to Fas or TRAIL mediated apoptosis [35-42], and renders them susceptible to immune 


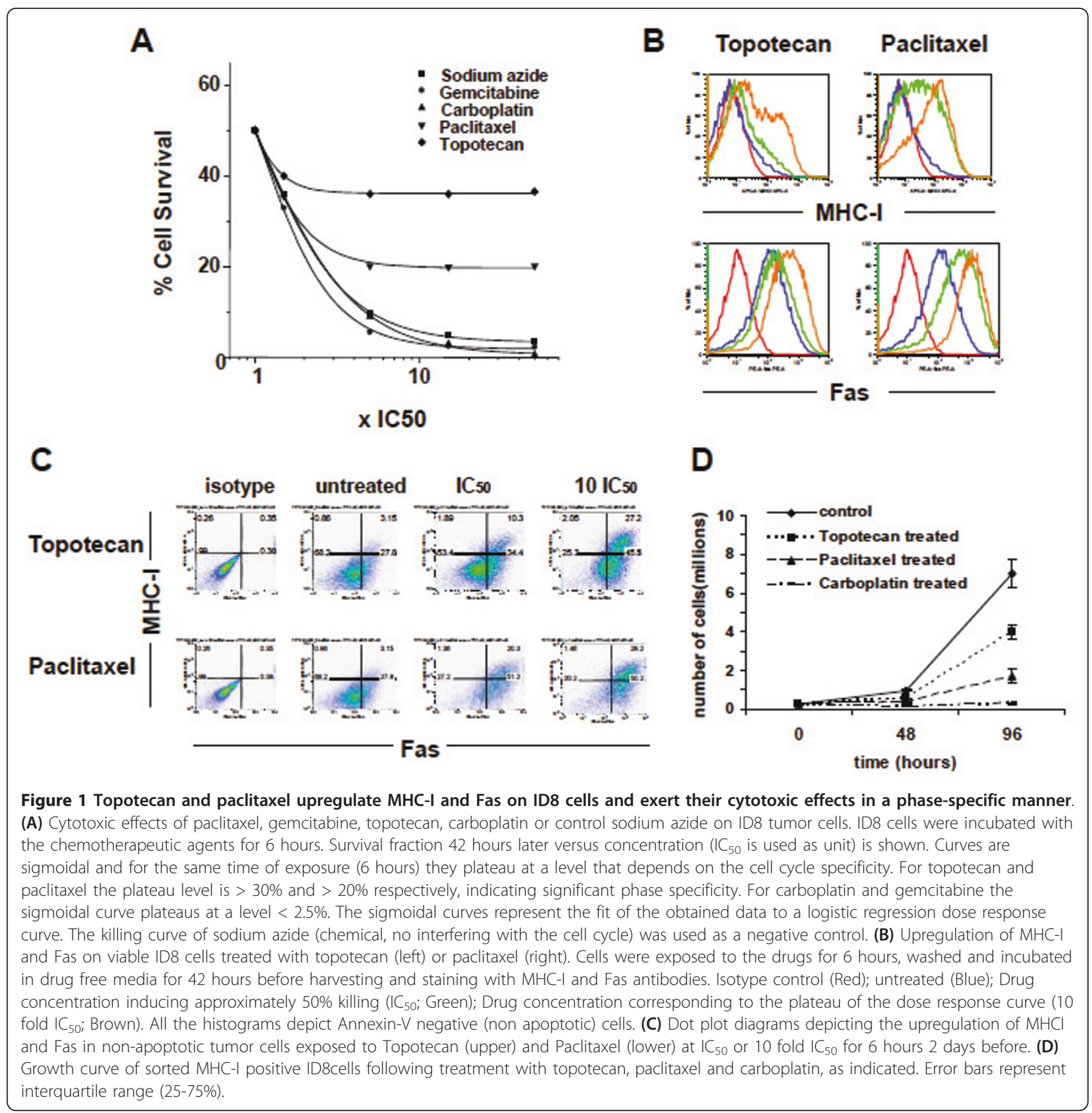

effector cells [3-6]. However, upregulation of immune molecules in tumor cells that have sustained lethal damage from chemotherapy and are destined to die regardless is unlikely to create a suitable basis for synergy between chemotherapy and immunotherapy. Rather, to generate positive interactions, immune effector mechanisms should be able to target tumor cells that are able to escape death from chemotherapy.

We examined whether cells that escape death from time-dependent drugs such as topotecan or paclitaxel upregulate immune molecules implicated in tumor immune attack. We measured the expression of MHC-I and death molecules Fas, TNF-related apoptosis-inducing ligand (TRAIL) and death receptor 6 (DR6) on ID8 cells after short exposure to topotecan or paclitaxel. Dead cells were identified by propidium iodide and apoptotic cells by annexin- $\mathrm{V}$ binding. Flow cytometry analysis 42 hrs following exposure to drug showed upregulation of MHC-I and Fas in a subset of viable (propidium iodide-negative and annexin V-negative) ID8 cells (Figure 1B). The upregulation of both MHC-I and Fas in viable cells was dose-dependent and was seen at $\mathrm{IC}_{50}$ 
as well as at concentrations corresponding to the plateau of killing curves (Figures 1B, C). The frequency of non-apoptotic ID8 cells co-expressing MHC-I and Fas after exposure to drugs at 10 -fold $\mathrm{IC}_{50}$ was seven-fold higher, compared to untreated control cells (27\% vs. < $4 \%$, respectively; Figure 1C). Similarly, a fraction of apoptotic (annexin- $\mathrm{V}$ positive) cells also upregulated MHC-I and Fas after drug exposure (data not shown). Non-apoptotic ID8 cells did not upregulate expression of the TRAIL receptor DR5, following exposure to paclitaxel or topotecan (not shown). Of note, ID8 cells exposed to carboplatin or gemcitabine also showed dose-dependent upregulation of MHC-I and Fas to a similar degree (not shown). These results show that short exposure to time-dependent (as well as dosedependent) drugs results in a population of tumor cells that could become potential targets for immune effector cells.

\section{MHC-I positive tumor cells are capable of re-expanding following exposure to phase-specific drugs}

Next, we examined the fate of tumor cells that upregulate MHC-I following short exposure to time-dependent or dose-dependent drugs. We asked whether these cells can undergo proliferation and restore tumor mass. ID8 cells were exposed to topotecan, paclitaxel, carboplatin or gemcitabine at $\mathrm{IC}_{70}$ for 6 hours and were then followed in drug-free conditions for 42 hours. Annexin-V negative, MHC-I positive ID8 cells were purified by FACS and were plated in normal media for four additional days. Cells exposed to topotecan proliferated in vitro and reached a tumor cell number that was approximately $60 \%$ that of a similar starting number of control ID8 cells that were never exposed to cytotoxic agents (Figure 1D). Cells exposed to paclitaxel also proliferated in vitro and reached approximately $20 \%$ of the number of control untreated ID8 cells (Figure 1D). Thus, following primary exposure to time-dependent drugs, a fraction of annexin- $\mathrm{V}$ negative cells that upregulate MHC-I remain viable and maintain their proliferative potential. Similar results were seen with the annexin-V negative, Fas-positive cells following exposure to topotecan or paclitaxel (data not shown). In contrast, annexin- $\mathrm{V}$ negative, MHC-I positive ID8 cells treated with carboplatin (Figure 1D) or gemcitabine at $\mathrm{IC}_{70}$ (not shown) exhibited no growth in vitro. Similarly, annexin$\mathrm{V}$ negative, Fas-positive ID8 cells treated with carboplatin or gemcitabine at $\mathrm{IC}_{70}$ exhibited no growth in vitro (not shown). Collectively, these results indicate that, although all tested drugs induce a population of tumor cells that expresses MHC-I and Fas and may be targeted by immune effector cells, this population remains viable and is able to restore tumor and thus biologically relevant, only in the case of phase-specific, time-dependent drugs. We thus hypothesized that combination with therapy that boosts immune effector cells benefits selectively time-dependent drugs but not dose-dependent drugs.

\section{Tumor cells surviving topotecan or paclitaxel are sensitized to effector $T$ cells}

To test the above hypothesis, we used as targets ID8E6E7 cells, a clonal population of ID8 cells retrovirally transfected to express HPV 16 E6 and E7 oncogenes [24]. ID8-E6E7 cells were treated with topotecan or paclitaxel for 6 hours at $\mathrm{IC}_{60}$ or a dose 10 fold the $\mathrm{IC}_{60}$ and were harvested $42 \mathrm{hrs}$ later to assess their susceptibility to immune-mediated killing. Effector cells reactive to HPV E7 were expanded from splenocytes of mice previously vaccinated against HPV E7 using E7 synthetic peptide. Prior exposure to paclitaxel or topotecan significantly increased killing of viable ID8-E6E7 cells by splenocytes relative to untreated cells (Figure 2A). Splenocytes from E7-vaccinated mice did not kill control ID8 cells that do not express E7, either at baseline or following exposure to topotecan or paclitaxel (not shown).

To assess whether chemotherapy-treated, viable (annexin-V negative) MHC-I positive and Fas-positive cells are also susceptible to Fas-mediated killing, treated cells were incubated with Fas agonistic antibody. Again, cells treated with time-dependent drugs paclitaxel or topotecan showed increased sensitivity to Fas-induced death (Figure 2B). We tested whether addition to Fas agonistic antibody to chemotherapy increased overall killing of tumor cells. Treatment of ID8 cells with Fas agonistic antibody in combination with paclitaxel or topotecan reduced the surviving fraction by nearly $50 \%$ at all concentrations of drug tested ( 1 to 50 fold the $\mathrm{IC}_{50}$ ), but did not improve carboplatin or gemcitabine treatment at drug concentrations above 10 fold the $\mathrm{IC}_{50}$ (Figure 2C). These results support a significant positive interaction between Fas ligation and phase-specific chemotherapeutic drugs.

\section{IL-18 improves the antitumor effect of time-dependent drugs}

Given the above results in vitro, we hypothesized that the addition of IL-18 could selectively increase the efficacy of time-dependent chemotherapeutic drugs such as topotecan or paclitaxel. First, we tested whether IL-18 exerts a direct cytotoxic effect on ID8 or ID8-Vegf tumor cells. No direct cytotoxic effect on ID8 or ID8Vegf tumor cells was seen in vitro (Figure $3 \mathrm{~A}$ and not shown).

Next, we assessed whether IL-18 therapy induces activation of effector $\mathrm{T}$ cells and restricts tumor growth in mice bearing orthotopic i.p. ID8-Vegf tumors. $\mathrm{CD}^{+}$ 


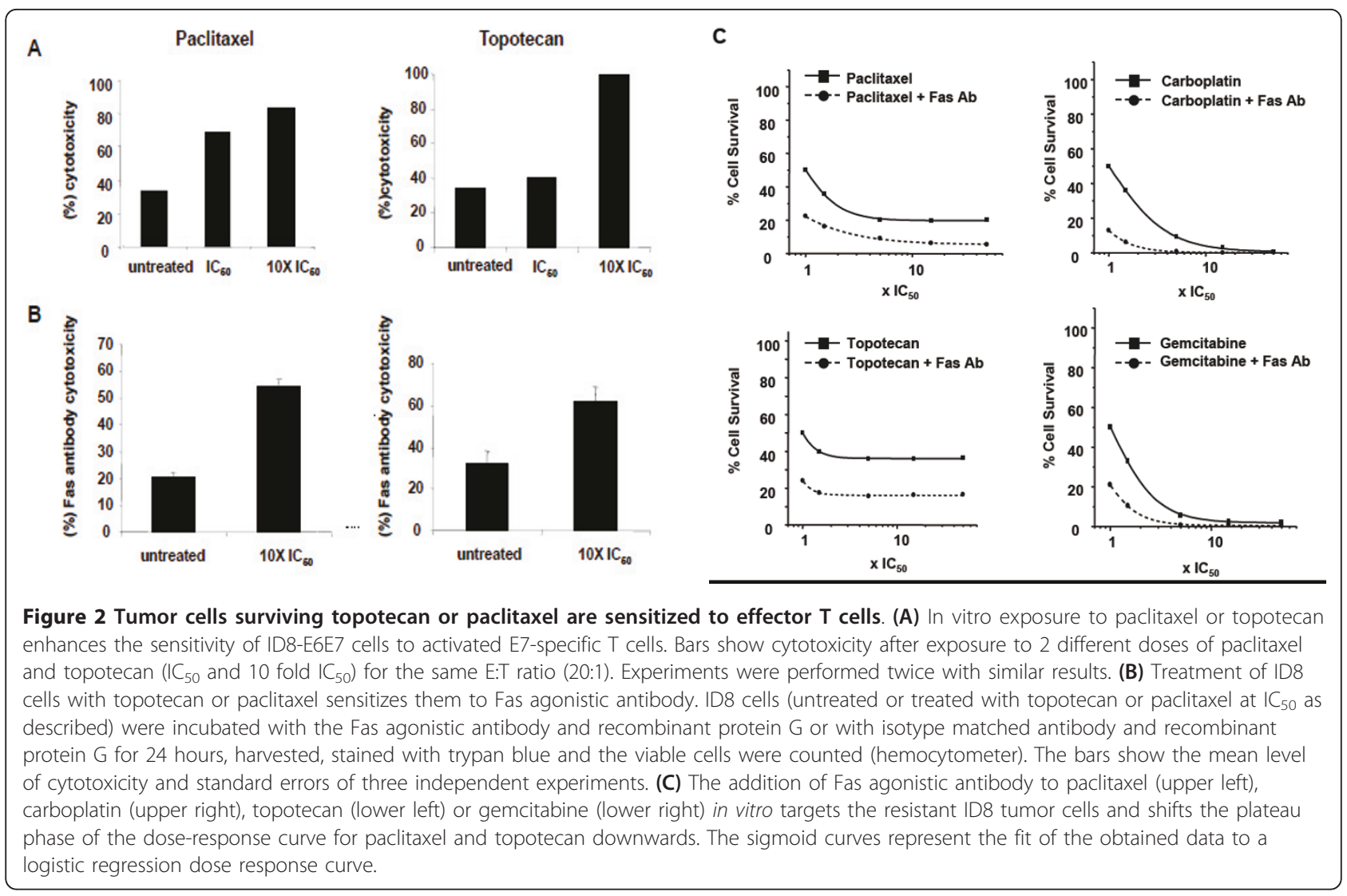

$\mathrm{CD}^{+}$splenocytes were isolated from mice bearing i.p. ID8-Vegf tumors and treated with $10 \mu \mathrm{g}$ of IL-18 daily for 20 days, starting 10 days following tumor inoculation. A $>2$ fold increase in the frequency of $\mathrm{CD}^{+} \mathrm{CD} 8$ + splenocytes expressing CD69 (12 $\pm 1.4 \%$ for saline $v$ s. $26 \pm 3.2 \%$ for IL-18) was seen after 20 days of IL-18 treatment (Figure 3B). Moreover, IL-18 therapy restricted ascites accumulation, a reliable surrogate of $\mathrm{i}$. p. tumor growth $(\mathrm{p}<0.05$, Figure $3 \mathrm{C})$, and prolonged the survival of ID8-Vegf tumor-bearing mice $(\mathrm{p}<0.05$; Figure 3D).

To assess the interactions between IL-18 and timedependent drugs, we treated animals bearing i.p. ID8Vegf tumors, with paclitaxel at $15 \mathrm{mg} / \mathrm{kg}$ (weekly, four doses), IL-18 (10 $\mu \mathrm{g} /$ day, 40 days), or paclitaxel plus IL18. Chemotherapy treatment was started 8 days following tumor inoculation and IL-18 treatment was started 2 days later. Control mice were treated with $0.9 \%$ saline alone. Paclitaxel alone prolonged median survival by 39\% (57 days) while IL-18 alone prolonged median survival by $17 \%$ ( 48 days; Figure $4 \mathrm{~A}$ ). In comparison with paclitaxel monotherapy, the combination of paclitaxel with IL-18 resulted in significant suppression of ascites accumulation $(\mathrm{p}<0.001)$ and better survival $(\mathrm{p}<0.01)$, prolonging median survival by $59 \%$ ( 65 days $v s$. 41 days in the control group). Survival at 100 days was $0 \%$ in the group treated with paclitaxel; $18 \%$ in group treated with IL-18; and $31 \%$ in the group treated with the combinatorial therapy; $(\mathrm{p}<0.01)$.

IL-18 administered as above yielded similar results in combination with topotecan at $2.5 \mathrm{mg} / \mathrm{kg}$ (every 5 days, 5 doses) (Figure 4B). The combination of IL-18 with topotecan prolonged median survival by $35 \%$ ( 58 day; $\mathrm{p}$ $<0.01$ relative to topotecan monotherapy), while IL-18 monotherapy prolonged median survival by $14 \%$ (49 days) and topotecan alone by $16 \%$ (50 days $v .43$ days in the control group; $\mathrm{p}<0.01$ ). The combination of IL-18 with topotecan also reduced ascites accumulation compared to topotecan monotherapy $(\mathrm{p}<0.001)$

\section{IL-18 does not improve the antitumor effect of dose- dependent drugs}

To reveal the significance of time-dependent drug properties in the interactions between IL-18 and chemotherapy, we tested the combinations of IL-18 with carboplatin or gemcitabine, two chemotherapeutic drugs that kill tumor cells in a dose-dependent manner. Carboplatin was given at $20 \mathrm{mg} / \mathrm{kg}$ (weekly, four doses), while gemcitabine was given at $25 \mathrm{mg} / \mathrm{kg}$ (every 3 days, 5 doses). IL-18 was given as above. Combination of IL- 


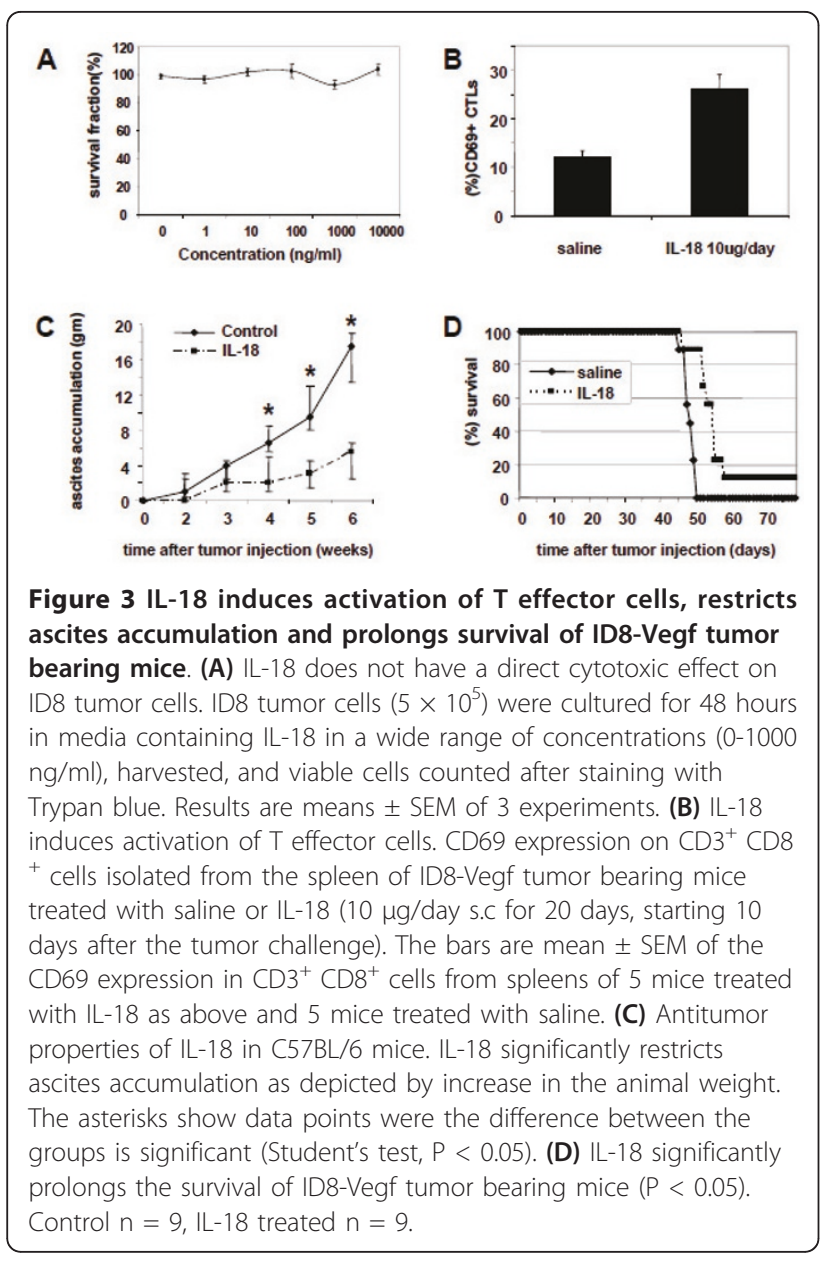

18 with carboplatin or with gemcitabine did not improve the effect of the chemotherapeutic agent alone either in terms of survival or in terms of ascites accumulation (Figure 4C, D). The median survival in the group treated with carboplatin alone was identical to the group that received the combination therapy. Similarly, the median survival afforded by the combination of IL-18 with gemcitabine was the same as gemcitabine alone. In both cases, IL-18 monotherapy modestly prolonged median survival by $14-15 \%$. There was no significant difference in ascites accumulation between the chemotherapy drugs alone and combination therapy. These results confirm that in vivo interactions of IL-18 seen with time-dependent chemotherapy drugs are not seen with dose-dependent drugs.

\section{Positive interactions between IL-18 and phase-specific chemotherapy drugs are mediated by $T$ cells}

Based on the in vitro experiments described above, we hypothesized that the observed interactions between IL18 and time-dependent chemotherapy drugs are mediated by effector $\mathrm{T}$ cells which are activated by IL-18 and attack tumor cells surviving the insult of chemotherapy. To test this hypothesis, we evaluated the combination of IL-18 with topotecan in SCID mice lacking T cells. ID8-Vegf cells were injected s.c. in the flank of immunocompetent C57BL/6 or SCID mice. Chemotherapy treatment was started 2 weeks after tumor inoculation, while IL-18 treatment was started 2 days after initiation of chemotherapy. In C57BL/6 mice, combination therapy decreased tumor growth relative to control mice $(\mathrm{p}<0.05)$, while IL-18 or topotecan alone did not have any significant effect (Figure 5A). The median tumor weight in the control group was $714 \mathrm{mg}$ (interquartile range 470-1278); in the topotecan group it was $419 \mathrm{mg}$ (325-540, $\mathrm{p}=0.06)$; in the IL-18 group it was $437.5 \mathrm{mg}(240-650, \mathrm{p}=0.066)$; and in the combination group it was $267 \mathrm{mg}(160-360, \mathrm{p}=0.012)$. In SCID mice, the effect of combination therapy was lost and there were no significant differences between the four experimental arms. The median and interquartile range in the control group were $631 \mathrm{mg}$ (476-962); in the topotecan group were $560 \mathrm{mg}(392-742, \mathrm{p}=0.4)$; in the IL-18 group were $463 \mathrm{mg}(317-840, \mathrm{p}=0.14)$; and in the combination group were $513 \mathrm{mg}$ (292-610, $\mathrm{p}=0.13$ ) (Figure $5 \mathrm{~B}$ ).

\section{Discussion}

Although chemotherapy is immunosuppressive and traditional assumption has been that chemotherapy negates the benefits of immunotherapy in cancer, evidence supporting the notion that chemotherapy can be associated with immunotherapy is mounting. For example, chemotherapy can potentially be enhanced by prior vaccine therapy [43]. However, it has been unclear to date whether any chemotherapy drugs are more suitable than others for such chemo-immunotherapy combinations. Whether chemo-immunotherapy combinations can significantly improve the survival benefit obtained with the conventional treatments is under continuing investigation, albeit primarily in empirically designed studies using dose-dependent agents [2,43-48]. Here we characterized the interactions of chemotherapy drugs used commonly against solid tumors with IL-18, a pleiotropic immunostimulatory cytokine. We did not provide direct evidence of phase-selective killing of ID 8 cells by the specific chemotherapeutic drugs in this study, because the mechanism of killing of these drugs has been widely documented in the literature so far, including with ovarian cancer cells. For example, in vivo and in vitro studies with topotecan, an S-phase specific drug, demonstrated that a high dose given by bolus injection was less effective than a lower dose given by continuous exposure $[49,50]$. Our findings indicate for the first time to our knowledge that chemotherapy drugs differ in their ability to interact with immunotherapy, depending on their mechanism of action. Time-dependent (phase- 
A

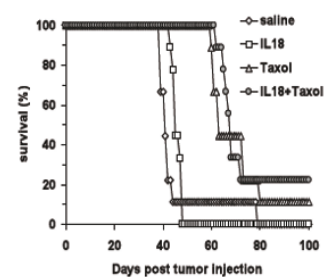

B

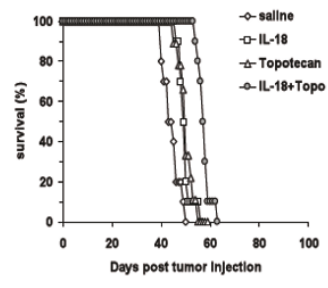

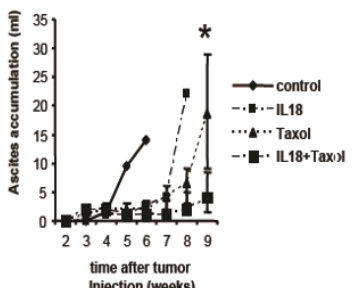

C

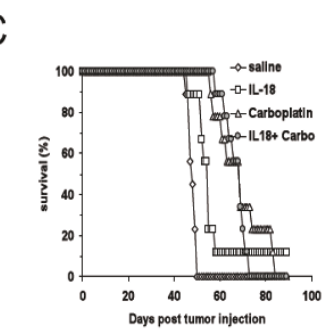

D
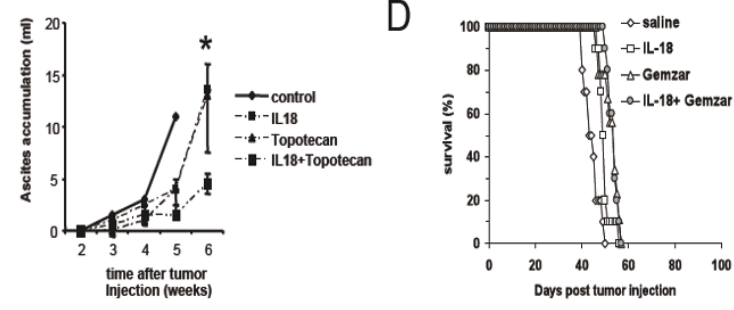
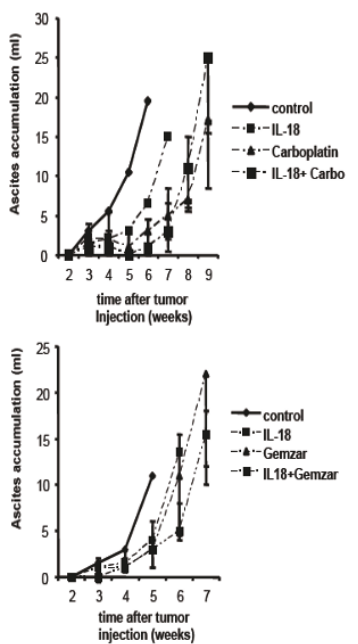

Figure 4 IL-18 improves only the antitumor effect of time-dependent drugs. (A) Combination therapy with IL-18 improves the survival benefit offered by paclitaxel and topotecan alone and restricts ascites accumulation (left). C57BL/6 mice were injected i.p. with $5 \times 10^{6}$ ID8-Vegf cells on day 0 and subsequently treated with the indicated types of treatment. Mice treated with the combination of paclitaxel plus IL-18 had a significantly prolonged survival compared to the respective monotherapies $(p<0.01$; control $n=23$, IL-18 alone $n=22$, paclitaxel $n=14$, IL-18 plus paclitaxel $n=13$ ). Combination therapy significantly restricted ascites accumulation (right) compared to the respective monotherapies as measured by the increase in the animal weight. The asterisks show data points where the difference in ascites accumulation is significant (Student's test, $\mathrm{P}<0.001$ ). (B) The combination of topotecan plus IL-18 also significantly prolonged the survival comparing to the respective monotherapies ( $p<0.01$; control $n=10, I L-18$ alone $n=10$, topotecan alone $n=9$, IL-18 plus paclitaxel $n=10$ ). Combination therapy significantly restricted ascites accumulation (right). The addition of IL-18 to carboplatin (C) or gemcitabine (D) did not significantly improve survival (left) or restrict ascites accumulation (right). IL-18/carboplatin experiment: Control $n=9$, IL-18 $n=9$, carboplatin $n=9$, IL-18 plus carboplatin $n=9$. IL-18/gemcitabine experiment: Control $n=10$, IL-18 $n=10$, gemcitabine $n=9$, IL-18 plus gemcitabine $n=10$.

specific) agents, such as topotecan or paclitaxel exhibited positive interactions with immunotherapy in vivo while dose-dependent (phase-nonspecific) drugs did not.

Time-dependent drugs target tumor cells in vulnerable phases of the cell cycle, and the fraction of tumor cells killed depends mainly on time of exposure rather than on drug dose $[8,10]$. Given that the time of in vivo exposure to chemotherapy drugs administered in conventional formulations is limited by their short half-life [35,36], a large fraction of tumor cells takes up drug but survives the insult. Our in vitro studies indicate that although these cells remain viable and can reconstitute the tumor, chemotherapy exposes a novel "Achilles' hill" on them. The fraction of viable tumor cells surviving time-dependent chemotherapy exposure showed increased expression of MHC-I and Fas and increased sensitivity to cytotoxic lymphocytes and Fas agonistic antibody. Although MHC-I and Fas were upregulated also in tumor cells treated with phase-nonspecific drugs such as carboplatin and gemcitabine, these cells were all apoptotic and had no tumorigenic potential. The central role of the immune system, particularly effector $\mathrm{T}$ cells, in expanding the efficacy of time-dependent chemotherapy was confirmed by the abrogation of the IL-18 and topotecan interaction in tumor-bearing SCID mice lacking $\mathrm{T}$ cells. We cannot exclude contribution of other effector cell types, but we expect $T$ cells to be a significant part of the positive interactions between phase-specific chemotherapy drugs and IL-18 in vivo in the mouse and in the human. Thus, an intact immune system is required to observe the therapeutic effects of topotecan and our studies provide novel insights in the complex interactions between chemotherapy and immune therapy. Although the notion that chemotherapy upregulates MHC-I and Fas on tumor cells is not novel, the notion that this becomes biologically and clinically relevant particularly for phase-specific drugs is novel and generates new opportunities for the rational design of chemo-immunotherapy combinations.

In this study we specifically focused on whether, and which, chemotherapy agents sensitize tumor cells to immune effector mechanisms; we thus tried to mainly explore the ability of drugs to render tumor cells more susceptible to immunotherapy with IL-18, and did not investigate each drug's overall effects on the immune system. Additional interactions are possible between chemotherapy and immune therapy. Cytotoxic drugs, when combined with immunotherapy, have the potential to provide a variety of agonistic effects to overcome the multiple barriers to natural antitumor responses. First, 


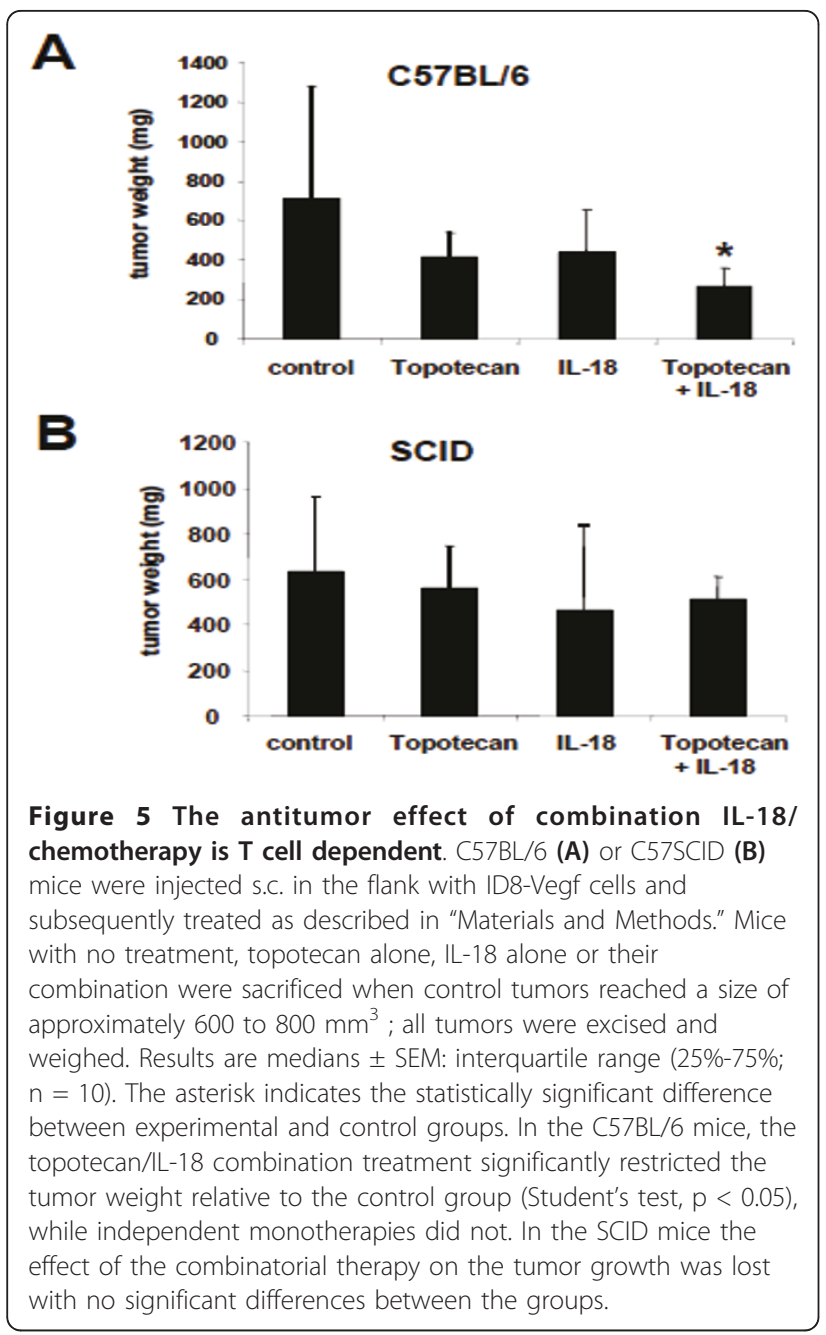

chemotherapy can diminish the impact of tumor immunoregulatory factors by reducing tumor burden. For example, during monitoring of $\mathrm{T}$ cell responses to antigenic epitopes of cytomegalovirus, EBV, and influenza in advanced ovarian carcinoma patients, it was found that $\mathrm{CD} 8^{+} \mathrm{T}$ cell responses were significantly lower in patients with high levels of CA125 than in those with low CA125 levels. Furthermore prospective monitoring before, during, and after first-line carboplatin/paclitaxel chemotherapy revealed that $\mathrm{CD} 8^{+} \mathrm{T}$ cell responses were restored by chemotherapy but only in patients in remission, while patients with progressive disease did not show improvement of $\mathrm{CD}^{+} \mathrm{T}$ cell responses [51]. In addition, chemotherapy can directly eliminate immunosuppressive cells such as regulatory $\mathrm{T}$ cells and/or myeloid suppressor cells; deplete host cells that compete with tumor antigen-specific $\mathrm{T}$ cells for homeostatic cytokines required for $\mathrm{T}$ cell proliferation and survival; and/or promote robust activation of professional antigen presenting cells [52]. Further, cytotoxic agents can modify the tumor microenvironment to favor antitumor responses through the promotion of tumor antigen processing and presentation, increased antigen uptake and improved homing of immune cells to tumor [53]. Specifically for gemcitabine, in addition to its apoptotic effects, it promotes the cell-mediated immune response over the humoral immune response by selectively inhibiting B-cell proliferation [54], decreasing memory $\mathrm{T}$ cells, and promoting the activation of naive $\mathrm{T}$ cells [55] and function of CD8+ $\mathrm{T}$ cells [56]. Immunopotentiation is also achieved in part by the inhibitory effects of gemcitabine on myeloid-derived suppressor cells [57]. These mechanisms were recently reviewed by us [58]. Lastly, chemotherapy can induce anti-angiogenic effects [59-61], which could potentially further enhance $\mathrm{T}$ cell homing to tumors.

Chemotherapy in ovarian cancer patients has been shown to temporarily reverse immunosuppression, and decrease the proportion of regulatory $\mathrm{T}$ cells. Likewise, it increases the percentage of IFN- $\gamma$ secreting $\mathrm{CD}^{+}$cells 12-14 days after administration, thus offering a "window" period for the use of immunotherapy, maximizing the tumor killing effect of both modalities [62]. On the other hand, simultaneous administration of immunotherapy combined with standard chemotherapy, could also be beneficial to ovarian cancer patients and better than a week-delayed schedule [63]. Thereby, not only the choice of the active drug but also the timing of immunotherapy is crucial for optimal results and this should be elucidated in future studies. The observed lack of interaction of gemcitabine with IL-18 in this study could be attributed, not only to the drug's mechanism of action, but also to the specific pharmacologic properties of IL-18. Our results could be affected by the relative sensitivity of tumor cells to each chemotherapy drug, and tumors with increased sensitivity could show different interactions. However, increased sensitivity to the chemotherapy drug does not necessarily mean that interactions with IL-18 would be more pronounced. Similarly, reduced sensitivity to the drug should not preclude interactions to be seen.

\section{Conclusions}

Our results support the concept that immunotherapy can be used to increase the killing effect of chemotherapy. Tumor immunotherapy with IL-18 can significantly augment the killing fraction of phase-specific chemotherapeutic drugs and provide survival benefit. Given that phase specificity is an inherent characteristic of several chemotherapeutic drugs used in a variety of cancers and a major factor that leads to treatment failures, the potential use of immunotherapy as a means to increase the killing fraction of phase dependent drugs, especially in tumors with low mitotic fraction, needs clinical 
testing. The safety profile of IL-18 and its positive interactions with select anticancer chemotherapeutic agents strongly supports the clinical investigation of this combinatorial approach. Additionally, in order to improve the positive interaction effect of IL-18 and phase-specific chemotherapeutic drugs, different dosing schedules and ways of drug delivery (e.g. liposomal formulations, protein-bound particles, or nanoparticles) should be used in future studies.

\section{Acknowledgements and Funding}

This study was supported by research funding from GlaxoSmithKline and NCI P01-CA83638 SPORE in Ovarian Cancer grant.

\section{Author details}

'Ovarian Cancer Research Center, School of Medicine, University of Pennsylvania, Philadelphia, USA. ${ }^{2}$ Abramson Family Cancer Research Institute, School of Medicine, University of Pennsylvania, Philadelphia, USA. ${ }^{3}$ Department of Pathology and Laboratory Medicine School of Medicine, University of Pennsylvania, Philadelphia, USA. ${ }^{4}$ GlaxoSmithKline, King of Prussia, USA.

\section{Authors' contributions}

IA carried out the in vitro and in vivo studies and drafted portions of the manuscript. AF, CC, FB and SA contributed materials and assisted in various in vitro and vivo experiments. MT helped in manuscript revision and discussion. ZJ and CHJ participated in the design of the study and data discussion and interpretation. DJP helped to draft the manuscript and supervised statistical analyses. GC designed the study, supervised all steps in execution and wrote the manuscript. All authors read and approved the final manuscript.

\section{Competing interests}

The authors declare that they have no competing interests.

Received: 23 November 2010 Accepted: 25 May 2011

Published: 25 May 2011

\section{References}

1. Correale P, Cusi MG, Tsang KY, Del Vecchio MT, Marsili S, Placa ML, Intrivici C, Aquino A, Micheli L, Nencini C, et al: Chemo-immunotherapy of metastatic colorectal carcinoma with gemcitabine plus FOLFOX 4 followed by subcutaneous granulocyte macrophage colony-stimulating factor and interleukin-2 induces strong immunologic and antitumor activity in metastatic colon cancer patients. J Clin Oncol 2005, 23(35):8950-8.

2. Bajetta E, Del Vecchio M, Nova P, Fusi A, Daponte A, Sertoli MR, Queirolo P, Taveggia P, Bernengo MG, Legha SS, et al: Multicenter phase III randomized trial of polychemotherapy (CVD regimen) versus the same chemotherapy (CT) plus subcutaneous interleukin-2 and interferonalpha2b in metastatic melanoma. Ann Oncol 2006, 17(4):571-7.

3. Gasser S, Orsulic S, Brown EJ, Raulet DH: The DNA damage pathway regulates innate immune system ligands of the NKG2D receptor. Nature 2005, 436(7054):1186-90

4. Gasser S, Raulet DH: The DNA damage response arouses the immune system. Cancer Res 2006, 66(8):3959-62.

5. Groh V, Bahram S, Bauer S, Herman A, Beauchamp M, Spies T: Cell stressregulated human major histocompatibility complex class I gene expressed in gastrointestinal epithelium. Proc Natl Acad Sci USA 1996, 93(22):12445-50

6. Ohtsukasa S, Okabe S, Yamashita H, Iwai T, Sugihara K: Increased expression of CEA and MHC class I in colorectal cancer cell lines exposed to chemotherapy drugs. J Cancer Res Clin Oncol 2003, 129(12):719-26.
7. Durand RE, Vanderbyl SL: Tumor resistance to therapy: a genetic or kinetic problem? Cancer Commun 1989, 1(5):277-83.

8. Gardner SN: A mechanistic, predictive model of dose-response curves for cell cycle phase-specific and -nonspecific drugs. Cancer Res 2000, 60(5):1417-25.

9. Gieseler F, Rudolph P, Kloeppel G, Foelsch UR: Resistance mechanisms of gastrointestinal cancers: why does conventional chemotherapy fail? Int J Colorectal Dis 2003, 18(6):470-80.

10. Levasseur LM, Slocum HK, Rustum YM, Greco WR: Modeling of the timedependency of in vitro drug cytotoxicity and resistance. Cancer Res 1998, 58(24):5749-61.

11. Gracie JA, Robertson SE, McInnes IB: Interleukin-18. J Leukoc Biol 2003, 73(2):213-24.

12. Jonak ZL, Trulli S, Maier C, McCabe FL, Kirkpatrick R, Johanson K, Ho YS, Elefante L, Chen YJ, Herzyk D, et al: High-dose recombinant interleukin-18 induces an effective Th1 immune response to murine MOPC-315 plasmacytoma. J Immunother 2002, 25(Suppl 1):S20-7.

13. Herzyk DJ, Soos JM, Maier CC, Gore ER, Narayanan PK, Nadwodny KL, Liu S, Jonak ZL, Bugelski PJ: Immunopharmacology of recombinant human interleukin-18 in non-human primates. Cytokine 2002, 20(1):38-48.

14. Robertson MJ, Mier JW, Logan T, Atkins M, Koon H, Koch KM, Kathman S, Pandite LN, Oei C, Kirby LC, et al: Clinical and biological effects of recombinant human interleukin-18 administered by intravenous infusion to patients with advanced cancer. Clin Cancer Res 2006, 12(14 Pt 1):4265-73.

15. Robertson MJ, Kirkwood JM, Logan TF, Koch KM, Kathman S, Kirby LC, Bell WN, Thurmond LM, Weisenbach J, Dar MM: A dose-escalation study of recombinant human interleukin-18 using two different schedules of administration in patients with cancer. Clin Cancer Res 2008, 14(11):3462-9.

16. Horwitz SB: Taxol (paclitaxel): mechanisms of action. Ann Oncol 1994, 5(Suppl 6):S3-6.

17. Lorusso D, Pietragalla A, Mainenti S, Masciullo V, Di Vagno G, Scambia G: Review role of topotecan in gynaecological cancers: current indications and perspectives. Crit Rev Oncol Hematol 2010, 74(3):163-74.

18. Duffull SB, Robinson BA: Clinical Pharmacokinetics and Dose Optimisation of Carboplatin. Clin Pharmacokinet 1997, 33(3):161-83.

19. Rockwell S, Grindey GB: Effect of 2',2'-difluorodeoxycytidine on the viability and radiosensitivity of EMT6 cells in vitro. Oncol Res 1992, 4(45):151-5.

20. Ruiz van Haperen WW, Veerman G, Vermorken JB, Peters GJ: 2',2'-Difluorodeoxycytidine (gemcitabine) incorporation into RNA and DNA of tumour cell lines. Biochem Pharmacol 1993, 46(4):762-6.

21. Mini E, Nobili S, Caciagli B, Landini I, Mazzei T: Cellular pharmacology of gemcitabine. Ann Oncol 2006, 17(Suppl 5):V7-12.

22. Zhang L, Yang N, Garcia JR, Mohamed A, Benencia F, Rubin SC, Allman D, Coukos G: Generation of a syngeneic mouse model to study the effects of vascular endothelial growth factor in ovarian carcinoma. Am J Pathol 2002, 161(6):2295-309.

23. Roby KF, Taylor CC, Sweetwood JP, Cheng Y, Pace JL, Tawfik O, Persons DL, Smith PG, Terranova PF: Development of a syngeneic mouse model for events related to ovarian cancer. Carcinogenesis 2000, 21(4):585-91.

24. Benencia F, Courreges MC, Coukos G: Whole tumor antigen vaccination using dendritic cells: comparison of RNA electroporation and pulsing with UV-irradiated tumor cells. J Trans/ Med 2008, 6:21.

25. Halbert CL, Demers GW, Galloway DA: The E7 gene of human papillomavirus type 16 is sufficient for immortalization of human epithelial cells. Journal of virology 1991, 65(1):473-8.

26. Bergman AM, Eijk PP, Ruiz van Haperen WW, Smid K, Veerman G, Hubeek I, van den ljssel P, Ylstra B, Peters GJ: In vivo induction of resistance to gemcitabine results in increased expression of ribonucleotide reductase subunit M1 as the major determinant. Cancer Res 2005, 65(20):9510-6.

27. De Cesare M, Zunino F, Pace S, Pisano C, Pratesi G: Efficacy and toxicity profile of oral topotecan in a panel of human tumour xenografts. Eur J Cancer 2000, 36(12):1558-64.

28. Mangold G, Dexter D, Juniewicz P, Rake J, Von Hoff D: Evaluation of paclitaxel-carboplatin-tirapazamine combinations in MV-522 human lung carcinoma xenograft model. ASCO Annual Meeting 1997, Abstract.

29. Travis EL, Bucci L, Fang MZ: Residual damage in mouse lungs at long intervals after cyclophosphamide treatment. Cancer Res 1990, 50(7):2139-45. 
30. Wiedenmann N, Valdecanas D, Hunter N, Hyde S, Buchholz TA, Milas L, Mason KA: 130-nm albumin-bound paclitaxel enhances tumor radiocurability and therapeutic gain. Clin Cancer Res 2007, 13(6):1868-74.

31. Euhus DM, Hudd C, LaRegina MC, Johnson FE: Tumor measurement in the nude mouse. J Surg Oncol 1986, 31(4):229-34.

32. Peng $X$, Treml J, Patterson $Y$ : Adjuvant properties of listeriolysin $O$ protein in a DNA vaccination strategy. Cancer Immunol Immunother 2007, 56(6):797-806.

33. O'Dwyer PJ, LaCreta FP, Haas NB, Halbherr T, Frucht H, Goosenberg E, Yao KS: Clinical, pharmacokinetic and biological studies of topotecan. Cancer Chemother Pharmacol 1994, 34(Suppl):S46-52.

34. Walle T, Walle UK, Kumar GN, Bhalla KN: Taxol metabolism and disposition in cancer patients. Drug Metab Dispos 1995, 23(4):506-12.

35. Ciusani E, Croci D, Gelati M, Calatozzolo C, Sciacca F, Fumagalli L, Balzarotti M, Fariselli L, Boiardi A, Salmaggi A: In vitro effects of topotecan and ionizing radiation on TRAIL/Apo2L-mediated apoptosis in malignant glioma. J Neurooncol 2005, 71(1):19-25.

36. Dejosez M, Ramp U, Mahotka C, Krieg A, Walczak H, Gabbert HE, Gerharz CD: Sensitivity to TRAIL/APO-2L-mediated apoptosis in human renal cell carcinomas and its enhancement by topotecan. Cell Death Differ 2000, 7(11):1127-36.

37. Mattarollo SR, Kenna T, Nieda M, Nicol AJ: Chemotherapy pretreatment sensitizes solid tumor-derived cell lines to V alpha 24+ NKT cellmediated cytotoxicity. Int J Cancer 2006, 119(7):1630-7.

38. Micheau O, Solary E, Hammann A, Martin F, Dimanche-Boitrel MT: Sensitization of cancer cells treated with cytotoxic drugs to fasmediated cytotoxicity. J Natl Cancer Inst 1997, 89(11):783-9.

39. Morimoto $H$, Yonehara S, Bonavida B: Overcoming tumor necrosis factor and drug resistance of human tumor cell lines by combination treatment with anti-Fas antibody and drugs or toxins. Cancer Res 1993, 53(11):2591-6

40. Straughn JM Jr, Oliver PG, Zhou T, Wang W, Alvarez RD, Grizzle WE, Buchsbaum DJ: Anti-tumor activity of TRA-8 anti-death receptor 5 (DR5) monoclonal antibody in combination with chemotherapy and radiation therapy in a cervical cancer model. Gynecol Oncol 2006, 101(1):46-54.

41. Tomek S, Horak P, Pribill I, Haller G, Rössler M, Zielinski CC, Pils D, Krainer M: Resistance to TRAIL-induced apoptosis in ovarian cancer cell lines is overcome by co-treatment with cytotoxic drugs. Gynecol Oncol 2004, 94(1):107-14.

42. Yang S, Haluska FG: Treatment of melanoma with 5-fluorouracil or dacarbazine in vitro sensitizes cells to antigen-specific CTL lysis through perforin/granzyme- and Fas-mediated pathways. J Immunol 2004, 172(7):4599-608

43. Antonia SJ, Mirza N, Fricke I, Chiappori A, Thompson P, Williams N, Bepler G, Simon $\mathrm{G}$, Janssen $\mathrm{W}$, Lee $\mathrm{JH}$, et al: Combination of p53 cancer vaccine with chemotherapy in patients with extensive stage small cell lung cancer. Clin Cancer Res 2006, 12(3 Pt 1):878-87.

44. Atzpodien J, Kirchner H, Jonas U, Bergmann L, Schott H, Heynemann H, Fornara P, Loening SA, Roigas J, Müller SC, et al: Interleukin-2- and interferon alfa-2a-based immunochemotherapy in advanced renal cell carcinoma: a Prospectively Randomized Trial of the German Cooperative Renal Carcinoma Chemoimmunotherapy Group (DGCIN). J Clin Oncol 2004, 22(7):1188-94.

45. Ishida A, Miyazawa T, Miyazu Y, Iwamoto Y, Zaima M, Kanoh K, Sumiyoshi H, Doi M: Intrapleural cisplatin and OK432 therapy for malignant pleural effusion caused by non-small cell lung cancer. Respirology 2006, 11(1):90-7.

46. Kasamon YL, Flinn IW, Grever MR, Diehl LF, Garrett-Mayer E, Goodman SN Lucas MS, Byrd JC: Phase I study of low-dose interleukin-2, fludarabine, and cyclophosphamide for previously untreated indolent lymphoma and chronic lymphocytic leukemia. Clin Cancer Res 2005, 11(23):8413-7.

47. Massacesi C, Burattini L, Marcucci F, Bonsignori M: Short communication: the efficacy of fixed dose rate infusion of gemcitabine combined with IFN-alpha2a in patients with advanced refractory renal cell carcinoma. J Interferon Cytokine Res 2005, 25(3):165-8.

48. Parra HS, Tixi L, Latteri F, Bretti S, Alloisio M, Gravina A, Lionetto R, Bruzzi P, Dani C, Rosso R, et al: Combined regimen of cisplatin, doxorubicin, and alpha- $2 b$ interferon in the treatment of advanced malignant pleural mesothelioma: a Phase II multicenter trial of the Italian Group on Rare Tumors (GITR) and the Italian Lung Cancer Task Force (FONICAP). Cancer 2001, 92(3):650-6.
49. Hoskins P, Eisenhauer E, Beare S, Roy M, Drouin P, Stuart G, Bryson P, Grimshaw R, Capstick V, Zee B: Randomized phase II study of two schedules of topotecan in previously treated patients with ovarian cancer: a National Cancer Institute of Canada Clinical Trials Group study. J Clin Oncol 1998, 16(6):2233-7.

50. Burris H, Kuhn J, Johnson R, Marshall MH, Kuhn JG, Hilsenbeck SG, Von Hoff DD: Activity of topotecan, a new topoisomerase I inhibitor, against human tumor colony-forming units in vitro. J Natl Cancer Inst 1992, 84(23):1816-20.

51. Coleman S, Clayton A, Mason MD, Jasani B, Adams M, Tabi Z: Recovery of CD8+ T-cell function during systemic chemotherapy in advanced ovarian cancer. Cancer Res 2005, 65(15):7000-6.

52. Muranski P, Boni A, Wrzesinski C, Citrin DE, Rosenberg SA, Childs R, Restifo NP: Increased intensity lymphodepletion and adoptive immunotherapy-how far can we go? Nat Clin Pract Oncol 2006, 3(12):668-81.

53. Zitvogel L, Apetoh L, Ghiringhelli F, Kroemer G: Immunological aspects of cancer chemotherapy. Nat Rev Immunol 2008, 8(1):59-73.

54. Nowak AK, Robinson BW, Lake RA: Gemcitabine exerts a selective effect on the humoral immune response: implications for combination chemoimmunotherapy. Cancer Res 2002, 62(8):2353-8.

55. Plate JM, Plate AE, Shott S Bograd S, Harris JE: Effect of gemcitabine on immune cells in subjects with adenocarcinoma of the pancreas. Cancer Immunol Immunother 2005, 54(9):915-25.

56. Suzuki E, Kapoor V, Jassar AS, Kaiser LR, Albelda SM: Gemcitabine selectively eliminates splenic Gr-1+/CD11b+ myeloid suppressor cells in tumor-bearing animals and enhances antitumor immune activity. Clin Cancer Res 2005, 11(18):6713-21.

57. Bronte V, Apolloni E, Cabrelle A, Ronca R, Serafini P, Zamboni P, Restifo NP, Zanovello P: Identification of a CD11b(+)/Gr-1(+)/CD31(+) myeloid progenitor capable of activating or suppressing CD8(+) T cells. Blood 2000, 96(12):3838-46.

58. Kandalaft LE, Singh N, Liao JB, Facciabene A, Berek JS, Powell DJ Jr, Coukos G: The emergence of immunomodulation: combinatorial immunochemotherapy opportunities for the next decade. Gynecol Oncol 2010, 116(2):222-33.

59. Browder T, Butterfield CE, Kraling BM, Shi B, Marshall B, O'Reilly MS, Folkman J: Antiangiogenic scheduling of chemotherapy improves efficacy against experimental drug-resistant cancer. Cancer research 2000, 60(7):1878-86

60. Bocci G, Nicolaou KC, Kerbel RS: Protracted low-dose effects on human endothelial cell proliferation and survival in vitro reveal a selective antiangiogenic window for various chemotherapeutic drugs. Cancer research 2002, 62(23):6938-43.

61. Gasparini G: Metronomic scheduling: the future of chemotherapy? The lancet oncology 2001, 2(12):733-40.

62. Wu X, Feng QM, Wang Y, Shi J, Ge HL, Di W: The immunologic aspects in advanced ovarian cancer patients treated with paclitaxel and carboplatin chemotherapy. Cancer Immunol Immunother 2010, 59(2):279-91.

63. Braly P, Nicodemus CF, Chu C, Collins Y, Edwards R, Gordon A, McGuire W, Schoonmaker C, Whiteside T, Smith LM, et al: The immune adjuvant properties of front-line carboplatin-paclitaxel: a randomized phase 2 study of alternative schedules of intravenous oregovomab chemoimmunotherapy in advanced ovarian cance. J Immunother 2009, 32(1):54-65.

doi:10.1186/1479-5876-9-77

Cite this article as: Alagkiozidis et al:: Time-dependent cytotoxic drugs selectively cooperate with IL-18 for cancer chemo-immunotherapy. Journal of Translational Medicine 2011 9:77. 